\title{
Micromechanical model for protein materials: From macromolecules to macroscopic fibers
}

\author{
G. Puglisi and D. De Tommasi \\ Dipartimento di Scienze dell'Ingegneria Civile e dell'Architettura, Via Re David 200, 700126, Politecnico di Bari, Italy
}

\begin{abstract}
M. F. Pantano
Laboratory of Bio-Inspired and Graphene Nanomechanics, Department of Civil, Environmental and Mechanical Engineering, University of Trento, Via Mesiano 77, 38123 Trento, Italy
\end{abstract}

\author{
N. M. Pugno \\ Laboratory of Bio-Inspired and Graphene Nanomechanics, Department of Civil, Environmental and Mechanical Engineering, \\ University of Trento, Via Mesiano 77, 38123 Trento, Italy; \\ Ket Lab, Edoardo Amaldi Foundation, Italian Space Agency, Via del Politecnico snc, 00133 Rome, Italy; \\ and School of Engineering and Materials Science, Queen Mary University of London, Mile End Road, London E1 4NS, United Kingdom \\ G. Saccomandi \\ Dipartimento di Ingegneria, Universita degli Studi di Perugia, piazza Universita, 1, 06123 Perugia \\ and School of Mathematics, Statistics and Applied Mathematics, NUI Galway, University Road, Galway, Ireland
}

(Received 13 December 2016; published 11 October 2017)

\begin{abstract}
We propose a model for the mechanical behavior of protein materials. Based on a limited number of experimental macromolecular parameters (persistence and contour length) we obtain the macroscopic behavior of keratin fibers (human, cow, and rabbit hair), taking into account the damage and residual stretches effects that are fundamental in many functions of life. We also show the capability of our approach to describe the main dissipation and permanent strain effects observed in the more complex spider silk fibers. The comparison between our results and the data obtained experimentally from cyclic tests demonstrates that our model is robust and is able to reproduce with a remarkable accuracy the experimental behavior of all protein materials we tested.
\end{abstract}

DOI: 10.1103/PhysRevE.96.042407

\section{INTRODUCTION}

Experimental analyses [atomic force microscopy (AFM), optical and magnetic tweezers, nanoindentation] clearly show that the outstanding elasticity, toughness, strength, and selfhealing properties $[1,2]$ of protein materials originate from their secondary structure characterized by the presence of folded (crystal-like) domains, typically in the form of $\alpha$ helices or $\beta$ sheets, which can undergo unfolding as a consequence of an applied displacement. The efficacy of the unfolding mechanism is based on the presence of a large number of noncovalent forces [3], typically hydrogen-bonding, which act as sacrificial joints. Being much weaker than the covalent peptide bonds, these noncovalent interactions can easily break, causing unfolding phenomena $(\alpha \rightarrow \beta$ transition or $\beta$ domains unfolding). Such unfolding prevents the backbone fracture conciliating the typically conflicting request for high stiffness and toughness [4]. Indeed, the cooperative strength of noncovalent bonds provides proteins with stability and stiffness before unfolding begins, whereas the increase of the end-to-end length during unfolding is responsible for toughness properties.

The analysis of the energy, stability, and time scales regulating the mechanical unfolding of hard domains represents a complex and diffusely analyzed task that cannot be solved with available molecular dynamics approaches. In fact, these were demonstrated not to be able to reproduce experimental observations [5], such as hierarchical multi-scales interactions [4], nano confinement effects [6], asymmetric energy landscapes for folding and refolding effect [7].
Here, we assume, in a phenomenological approach, to know the experimental stretch induced unfolding behavior of the single macromolecule (see Fig. 2). Then, based on an (slightly amended) affinity hypothesis [8] and the James and Guth three-chains model [9], we deduce the macroscopic behavior with damage and residual stretch of protein materials and analytical relation with few key macromolecular material parameters. Specifically, as in Refs. [10] and [11], we consider for the single molecule under assigned elongation a phenomenological Griffith-type approach assuming that the macromolecule unfolds when the energy gain (evaluated as difference of the two force-elongation curves between which the macromolecule jumps) equals a material parameter, representing the energy "dissipated" in the mechanical unfolding of the crystal. The corresponding contour length (hidden length) variations of the macromolecules, which can be also evaluated by the macromolecular stretching experiment, allows to accommodate large stretches (20-40\%) with a contemporary high dissipation and toughness.

As in the case of multiscale dislocation and defect theories in metal plasticity [12], a significant advance in the comprehension of protein materials requires models relating the material properties at the macromolecular scale to the macroscopic response of fibers and tissues [2,10,13-16]. Such models are essential for both the comprehension of many biological (i.e., mechanotransduction and cell motility [17]) and biomedical phenomena (such as Alzheimers, type II Diabetes, Parkinsons [18]) and in the perspective of the design of new, high-performance bioinspired materials [2,13,19-24]. 


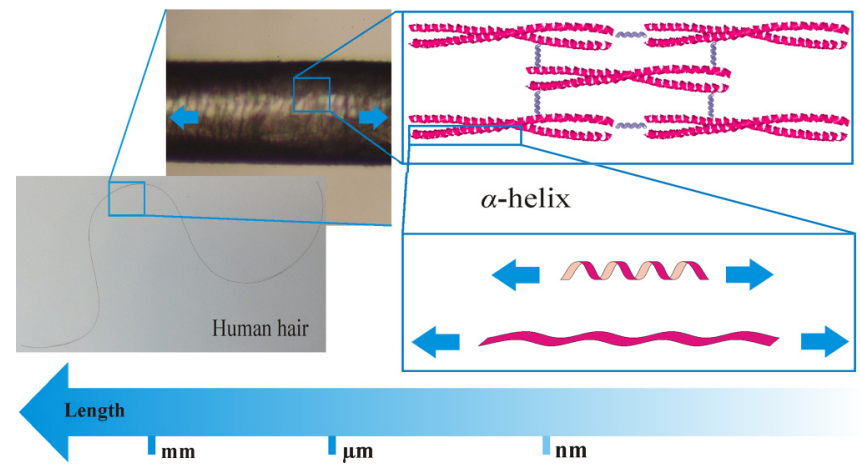

FIG. 1. Multiscale behavior of protein materials like human hair under stretching. When a hair is stretched, the $\alpha$-helix structures undergo a coiled-coil transition to an almost straight polypeptide chain in the form of $\beta$ sheets.

Here we focus on two different classes of structural protein materials [25] known for their outstanding performances: keratinous materials, which can be found in wool, hair, cells intermediate filaments, epithelial cells and hooves and silk, a valuable material produced by spiders and silkworms. In the first case, the secondary structure is in the form of intrachain $\alpha$ helices undergoing a coiled-coil transition to an almost straight polypeptide chain in the form of $\beta$ sheets $[13,26]$, whereas in the second case the protein secondary structure is in the form of interchains (alanine rich) $\beta$-sheet domains which undergo unfolding under stretching [14].

The $\alpha \rightarrow \beta$ transition is a diffusely adopted mechanism in many protein materials, such as cytoplasmic IF proteins [27], which needs deep comprehension as it is linked to many human deceases such as Alzheimer, prion diseases, Parkinson. Both silk and keratinous materials have attracted significant attention from the scientific literature for the design of new bioinspired materials among which we recall nanopolymers [22] and block copolymers [21] in the case of spider silk and hydrogels [23] in the case of keratinous materials.

\section{MICRO-MACRO MODEL}

The sketch of the multiscale behavior of a hair protein fiber is schematized in Fig. 1.

The main assumptions introduced to derive our multiscale model are the following (Fig. 3).

(a) Additive assumption. Following a classical approach in polymer elasticity [28] [Fig. 3(b)], the energy of the protein network is the sum of the energy of ideally noninteracting unfolding chains plus a term (here modeled as simple Gaussian chains) accounting for the real network chains interactions.

(b) Wang and Guth hypothesis. All macromolecules are aligned along the principal axes [Wang and Guth scheme [9], Fig. 3(b)] and undergo the macroscopic stretches (affinity hypothesis [8]). Since protein fibers (both silk [19] and keratin [29]) are produced as highly oriented filaments densely packed in a less-ordered matrix, macromolecule unfolding occurs along the fiber direction, whereas the amorphous soft fraction is equally distributed along the principal directions.

(c) Skin-core effect. The fiber behavior is strongly influenced by its skin-core structure ([30], supercontraction
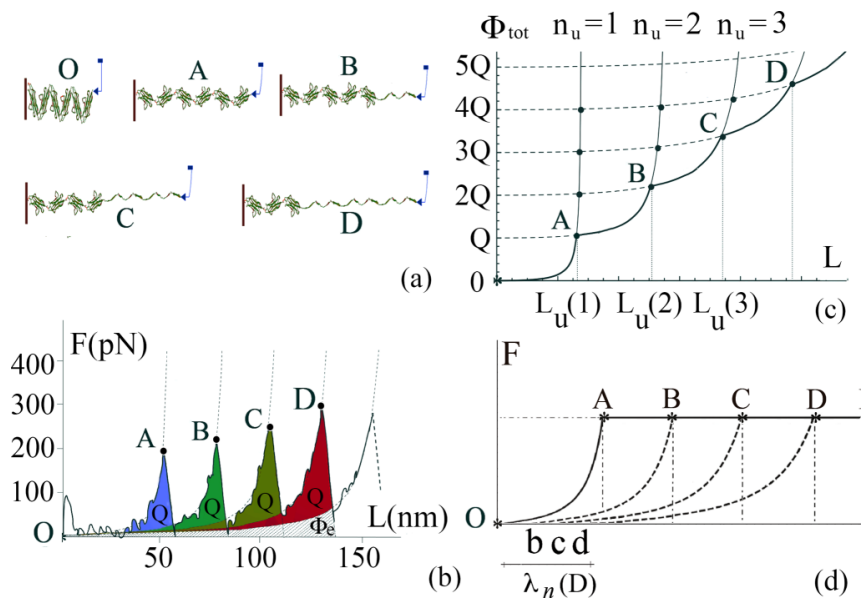

FIG. 2. (a) Scheme of second structure unfolding during an AFM molecule stretching and (b) corresponding force-elongation curve (experimental behavior reproduced from Ref. [34]). The sawtooth shape is due to unfolding of an increasing number of domains with (c) reporting the corresponding total energy minimization approach (see Ref. [11]). (d) Continuum limit approximation with constant unfolding force plateaux.

[19], confinement [31], and prestretch of the inner chains [32]) here considered by imposing on the fibers a transversal pressure $p$.

Remark. This scheme extends the model in Ref. [33] to consider anisotropic damage: only macromolecules along the fiber direction undergo unfolding. This is fundamental for the experimental effect of permanent stretches.

\section{A. Macromolecule energy}

AFM experiments [34], reproduced in Fig. 2(b), show that a protein macromolecule experiences, as schematized in Fig. 2(a), a sequence of periodic unfolding events as a consequence of an increasing applied displacement. This results in a contemporary entropy and contour length increase (hidden length: increased number of free monomers) accompanied by an energy "dissipation" ( $Q$ in the figure) due to $H$-bonds disruption.

Since typically no partial unfolding occurs (either all or none of the domains unfold) following a Griffith-type total energy minimization approach [11], as in Refs. [26] and
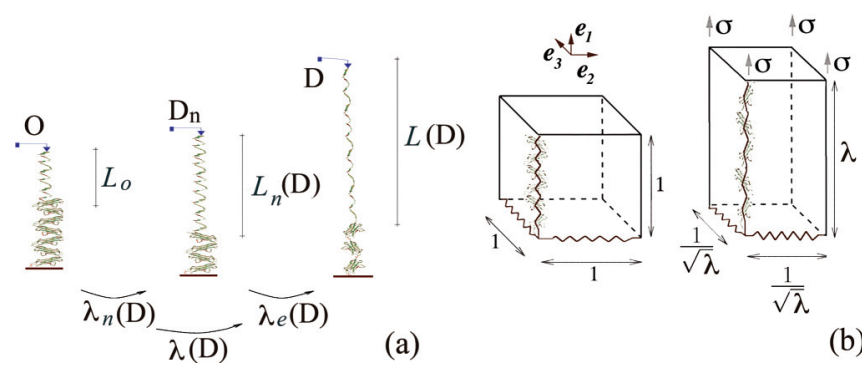

FIG. 3. (a) Cartoon showing the deformation induced chain unfolding, where the total stretch $\lambda$ is given by the product of the elastic stretch $\lambda_{e}$ and the permanent stretch $\lambda_{n}$. (b) Micro-macro multiscale network. 
[35], we model a protein macromolecule as a lattice of $n$ two-states (rigid-folded and entropic-unfolded) domains. We define the state of the $i$ th domain through the variable $\chi_{i}$ with $\chi_{i}=0(=1)$ in the folded (unfolded) state. For simplicity, since we are interested in the unfolding regime, we neglect both the elastic energy and the length of the folded domains. Moreover, we neglect nonlocal interactions (weak interaction hypothesis). The total internal energy can then be expressed as $\phi_{e}=\sum_{i=1}^{n} \chi_{i} \varphi_{e}\left(\lambda_{\text {rel }}^{i}\right)$, where $\varphi_{e}$ depends on the relative deformation $\lambda_{\text {rel }}^{i}=l_{i} / l_{c}$ of the $i$ th unfolded domain, with end-to-end length $l_{i}$, contour length (assumed constant) $l_{c}$ and on the persistent length $L_{p}$. We also assume the limit extensibility condition: $\lim _{\lambda_{\text {rel }} \rightarrow 1} \varphi_{e}\left(\lambda_{\text {rel }}\right)=+\infty$.

Equilibrium requires a constant force $l_{c} \frac{d \varphi_{e}\left(l_{i} / l_{c}\right)}{d l_{i}}=F$ and, under the hypothesis of convex $\varphi_{e}$, a constant $\lambda_{\text {rel }}^{i}=l_{i} / l_{c}=$ $L / L_{c}$. Here, $L=\sum_{i} \chi_{i} l_{i}$ is the total end-to-end length and $L_{c}=\sum_{i} \chi_{i} l_{c}$ is the total contour length of the unfolded fraction. As a result we have $\phi_{e}=L_{c} \varphi_{e}\left(\lambda_{\text {rel }}\right)=L_{c} \varphi_{e}\left(L / L_{c}\right)$. Here, for the energy of the unfolded fraction we adopt the Worm Like Chain (WLC) type entropic energy density proposed in Ref. [11] (this choice allows for analytic computations and keeps the same asymptotic behavior as $l \rightarrow l_{c}$ of the WLC model):

$$
\varphi_{e}=\varphi_{e}\left(l, l_{c}\right)=\kappa \frac{l^{2}}{l_{c}-l},
$$

where $\kappa=\frac{k_{B} T}{4 L_{p}}, T$ is the temperature and $k_{B}$ the Boltzmann constant. Thus, using the recalled strain homogeneity result in the entropic fraction, the total elastic energy is

$$
\phi_{e}=\kappa \frac{L^{2}}{L_{c}-L} .
$$

Let us consider now the configurational energy of the different folded and unfolded states. Following Ref. [11] (and references therein), here we consider an Ising-type transition energy,

$$
\begin{aligned}
\phi_{\mathrm{tr}} & =-\sum_{i=1}^{n}(Q-J)\left(1-\chi_{i}\right)-J \sum_{i=1}^{n-1}\left(1-\chi_{i}\right)\left(1-\chi_{i+1}\right) \\
& =-\sum_{i=1}^{n} Q\left(1-\chi_{i}\right)+J n_{b_{f}},
\end{aligned}
$$

where $n_{b_{f}}$ is the number of contiguous folded blocks in the folded and unfolded configuration, $Q$ is the unfolding energy of the folded domains (considered constant), and $J$ is a penalizing "interfacial" energy term. By introducing the probability function $\tilde{p}=\tilde{p}\left(L, n_{u}, n_{b_{f}}\right)$ of a state of the macromolecule with end-to-end length $L, n_{u}$ unfolded domains, and $n_{b_{f}}$ continuous folded blocks, the total energy can be expressed as

$$
\begin{aligned}
\phi_{\mathrm{tot}} & =-k_{B} T \ln \left[\tilde{p}\left(L, n_{u}, n_{b_{f}}\right)\right] \\
& =k_{B} T \Omega\left(n_{u}, n_{b_{f}}\right) \exp \left(-\frac{\phi_{e}\left(L, n_{u}\right)}{k_{B} T}\right) \exp \left(-\frac{\phi_{t r}\left(n_{u}\right)}{k_{B} T}\right) \\
& =\phi_{e}\left(L, n_{u}\right)+\phi_{\mathrm{tr}}\left(n_{u}\right)-T S\left(n_{u}, n_{b_{f}}\right),
\end{aligned}
$$

where $\Omega\left(n_{u}, n_{b_{f}}\right)$ is the number of sequences with assigned $n_{u}$ and $n_{b_{f}}$ and $S\left(n_{u}, n_{b_{f}}\right)=-k_{B} \ln \Omega\left(n_{u}, n_{b_{f}}\right)$ represents a mixing entropy component. By the diblock approximation, assuming always a single connected internal unfolded domain inside two boundary-folded domains $\left(n_{b_{f}}=1\right.$ as supported by the MD simulations in Ref. [41]), we neglect the mixing entropy term and the total (entropic plus unfolding) energy is simply

$$
\phi_{\text {tot }}=\phi_{e}\left(L, n_{u}\right)+n_{u} Q+\text { const, }
$$

where $\phi_{e}$ is given in Eq. (2).

In Figs. 2(b) and 2(c) we show the scheme of our minimization procedure, considering the global minimizer of $\phi_{\text {tot }}$ determining the lengths transition $L_{u}$ with an increasing number $n_{u}$ of unfolded domains: the system undergoes an unfolding transition as soon as the entropic energy gain equals the (enthalpic) unfolding energy $Q$ (colored area in the figure). The efficacy of this approach for the single chain has been evidenced in Ref. [11].

\section{B. Continuum macromolecule approximation}

To derive the macroscopic energy of a material consisting of a number of macromolecules, following Ref. [33] we begin by considering the continuum approximation of the macromolecular discrete lattice. To this end we fix the total unfolded length $L_{c}^{1}=n l_{c}$ and consider the (thermodynamic) limit when both $l_{c} \rightarrow 0$ and $n \rightarrow \infty$ (see Ref. [36] for a similar approach in the case of biological adhesion and Refs. [37] and [38] for a rigorous thermodynamical justification). After introducing the continuous variable $x \in\left(0, L_{c}^{1}\right)$ - such that the $i$ th link corresponds to $x \in\left(i l_{c},(i+1) l_{c}\right), i=1, \ldots n-$ and the dissipation density $q=Q / l_{c}$, the total energy for the continuum limit chain is

$$
\phi_{\mathrm{tot}}=\phi_{\mathrm{tot}}\left(L, L_{c}\right)=\kappa \frac{L^{2}}{L_{c}-L}+q L_{c} .
$$

The equilibrium force is given by

$$
f=\frac{\partial \phi_{\mathrm{tot}}\left(L, L_{c}\right)}{\partial L}=\kappa \frac{2 L L_{c}-L^{2}}{\left(L_{c}-L\right)^{2}},
$$

whereas the driving force $g$ conjugated to the variation of the contour length $L_{c}$ (i.e. with the percentage of unravelled domains) is given by

$$
g=-\frac{\partial \phi_{\mathrm{tot}}\left(L, L_{c}\right)}{\partial L_{c}}=\kappa \frac{L^{2}}{\left(L_{c}-L\right)^{2}}-q .
$$

The Griffith approach fixes the dissipation rate $g=\hat{g}$, where $\hat{g}$ is a given material parameter here assumed null (global energy minimization). This provides for given $L_{c}$ the unfolding length

$$
L_{\mathrm{un}} \equiv L_{\max }=\frac{L_{c}}{\sqrt{k / q}+1},
$$

and a constant unfolding force [path OABCD in Fig. 2(d)],

$$
f_{\text {un }}=(2 \sqrt{k / q}+1) q .
$$

Remark. Dashed lines in Fig. 2(d) are unloading and reloading paths. The introduction of partial refolding upon unloading would let us describe also internal hysteric effects. Similarly, the different behavior for macromolecular high rate of loading [7] could introduce rate loading effects [39]. 


\section{Affinity hypothesis}

As classical in polymer elasticity [8], the main hypothesis for the deduction of the macroscopic behavior is the affinity hypothesis, which assumes that the macroscopic stretches coincide with the macromolecular ones. We here give an interpretation of the permanent deformations observed in protein materials as a macroscopic counterpart of the variation of the macromolecule natural configuration associated with the unfolding of crystal domains and the availability of new monomers (hidden length). We then extend the affinity hypothesis by identifying both permanent and elastic components of the macromolecular stretch with the macroscopic ones. In such a way we are also able to describe the important permanent stretch effect.

To analytically describe this phenomenon we begin by observing that for a given contour length $L_{c}$ the natural length (zero force) $L_{n}$ of the entropic chain can be expressed, according to a known result of statistical mechanics (see, e.g., Ref. [8]), as

$$
L_{n}=\sqrt{\bar{n}} b=\sqrt{\frac{L_{c}}{b}} b=\sqrt{L_{c} b},
$$

where $b$ is the length of the Kuhn segments and $\bar{n}$ is the number of Kuhn segments of the unfolded chain fraction. Thus, if we denote by $L_{c}^{o}$ the initial value of the contour length, the initial natural end-to-end length is $L_{o}=\sqrt{L_{c}^{o} b}$. Then we may define the following stretch measures:

$$
\begin{aligned}
\lambda & =\frac{L}{L_{o}}, \quad \text { total stretch, } \\
\lambda_{e} & =\frac{L}{L_{n}}, \quad \text { elastic stretch, } \\
\lambda_{n} & =\frac{L_{n}}{L_{o}} \quad \text { permanent stretch, } \\
\lambda_{c} & =\frac{L_{c}}{L_{o}} \quad \text { limit extensibility stretch. }
\end{aligned}
$$

The continuum limit behavior with permanent stretches is illustrated in Fig. 2(d). Under assigned growing end-to-end length $\left(\lambda_{\max } \equiv \lambda\right.$ and $\left.\dot{\lambda}>0\right)$ the macromolecule stretches elastically until the threshold $f=f_{\text {un }}$ is attained and the macromolecule unfolds along a stress plateaux (path O-A-E). If the system is unloaded $\left(\lambda<\lambda_{\max }\right.$ and $\left.\dot{\lambda}_{\max }=0\right)$, then the system follows different paths (paths $\mathrm{Bb}, \mathrm{Cc}, \mathrm{Dd}$ ), with permanent stretches growing with $\lambda_{\text {max }}$.

We then obtain, using Eq. (5), the force-stretch relation

$$
f=\kappa \frac{\frac{2 \lambda}{\bar{\lambda}_{c}\left(\lambda_{\max }\right)}-\left[\frac{\lambda}{\bar{\lambda}_{c}\left(\lambda_{\max }\right)}\right]^{2}}{\left[1-\frac{\lambda}{\bar{\lambda}_{c}\left(\lambda_{\max }\right)}\right]^{2}},
$$

where $\lambda_{\max }$ is the previously maximum attained strain and, by using Eq. (7),

$$
\lambda_{c}=\bar{\lambda}_{c}\left(\lambda_{\max }\right)=\left(\sqrt{\frac{\kappa}{\bar{q}}}+1\right) \lambda_{\max },
$$

is the (history-dependent) value of the limit stretch corresponding to the attainment on the contour length deducible analytically by the present value of $\lambda_{\max }$.

\section{Macrosopic material behavior}

Structural proteins are composite materials of hard segments immersed in an amorphous soft fraction of unfolded macromolecules. To describe the complex interchains interactions and self-avoiding effects, according to the additivity hypothesis in polymer elasticity [28], we determine the energy of the real network as the sum of the energy of ideally isolated fibrils [folded molecules in Fig. 3(b), whose behavior is schematized in Fig. 2(d)] plus a network energy term measuring the chains interactions: red springs in Fig. 3(b). Moreover, according to the classical James and Guth threechains model, we assume that all chains are aligned along the three principal macroscopic stretch directions. In particular, we suppose that the unfolding protein macromolecules are oriented along the fiber direction, whereas the amorphous soft fraction is supposed to be equally distributed along the three principal directions. For the unfolding chains we assume the continuous limit energy Eq. (4), force Eq. (5), unfolding force Eq. (7), and stretches Eq. (8) deduced above. The network effect is instead modeled by simple Gaussian chains.

Thus, let $\left(\lambda_{1}, \lambda_{2}, \lambda_{3}\right)$ be the macroscopic principal stretches, where $\lambda_{1}$ is the stretch in the fiber direction. According to previous hypotheses, the macroscopic energy density can be calculated as

$$
\Phi\left(\lambda_{i}\right)=N_{\text {fib }} \phi_{\text {tot }}\left(\lambda_{1}\right)+\sum_{i=1}^{3} \frac{N_{\text {net }}}{3} \phi_{\text {net }}\left(\lambda_{i}\right) .
$$

Here $N_{\text {fib }}$ is the number of macromolecules with unfolding domain per unit area of fiber section in the reference configuration and $N_{\text {net }}$ is the number of chains, per unit volume, reproducing the real chains network effect. The network energy is modeled as Gaussian $\phi_{\text {net }}\left(\lambda_{i}\right)=\frac{k_{B} T}{2}\left(\lambda_{i}^{2}-1\right)$ leading (see, e.g., Ref. [8]) to a neo-Hookean network energy $\Phi_{\text {net }}=\frac{\mu}{2}(I-3)$, where $I=\sum_{i=1}^{3} \lambda_{i}^{2}$ is the first invariant of the left Cauchy-Green deformation tensor and $\mu=k_{B} T N_{\text {net }} / 3$ represents the shear elastic modulus. On the other hand, the energy of the unfolding chains gives a history dependent term depending only on $\lambda_{1}$ stretch. In such a way we describe a fundamental effect with the damage localized along the (maximum elongation) fiber direction. It is worth noticing that this damage anisotropy is crucial for an effective derivation of the macroscopic permanent stretch.

Suppose now that the fiber (assumed incompressible) undergoes uniaxial extension $\lambda_{1}=\lambda$ and $\lambda_{2}=\lambda_{3}=1 / \sqrt{\lambda}$. To take into account the confinement effect, we introduce a constant pressure $p<0$ perpendicular to the fiber skin. Thus, if $\sigma=\sigma_{1}$ is the (Piola or engineering) principal stress in the fiber direction, by using Eqs. (5) and (8) and by imposing the boundary conditions $\sigma_{2}=\sigma_{3}=p$, we get the stress-stretch relation,

$$
\begin{aligned}
\sigma= & N^{\mathrm{fib}} \kappa \frac{\frac{2 \lambda}{\bar{\lambda}_{c}\left(\lambda_{\max }\right)}-\left(\frac{\lambda}{\bar{\lambda}_{c}\left(\lambda_{\max }\right)}\right)^{2}}{\left(1-\frac{\lambda}{\bar{\lambda}_{c}\left(\lambda_{\max }\right)}\right)^{2}} \\
& +\mu\left(\lambda-\frac{1}{\lambda^{2}}\right)+\frac{p}{\lambda \sqrt{\lambda}},
\end{aligned}
$$

where $\lambda_{\max }$ is the previously maximum attained strain with $\lambda_{\max }=\lambda$ if $\dot{\lambda}_{\max }>0$ (primary loading) and $\lambda_{\max }=\mathrm{const}$ 
if $\dot{\lambda}_{\max }=0$ (unloading). Moreover, by using Eq. (7),

$$
\lambda_{c}=\bar{\lambda}_{c}\left(\lambda_{\max }\right)=\left(\sqrt{\frac{\kappa}{\bar{q}}}+1\right) \lambda_{\max }
$$

is the (limit) value of the stretch corresponding to the present value of the contour length (unloading and reloading).

\section{EXPERIMENTAL VALIDATION}

To prove the efficacy of our model, we performed cyclic tensile tests on two different types of material: keratin hair materials and dragline silk.

We first consider keratinous materials, such as human, cow, and rabbit hairs. The tests and the possibility of reproducing the macroscopic behavior of these keratin fibers are shown in Fig. 4.

The macromolecular parameters are coherent with the ones known in the literature. The values of the persistent length match those reported in Ref. [13], where $l_{p}=0.4 \mathrm{~nm}$. The values of the rate of dissipation $q=45 k_{B} T / \mathrm{nm}$ are coherent with the value in Ref. [27] from which one deduces that the energy for the unfolding of a single amino acid is about $100 \mathrm{kcal} / \mathrm{mol}$, which corresponds to $q=59.6 k_{B} T / \mathrm{nm}$, by assuming an amino acid length of about $1 \mathrm{~nm}$. A higher value $q=180 k_{B} T / \mathrm{nm}$ is adopted for the rabbit hair and this is due to the much higher experimental unfolding stress (about $600 \mathrm{MPa}$ ) that we here interpret as a particularly high rate of dissipation $q$ corresponding to higher energy barrier regulating the transition between the folded and unfolded configuration. The number $N=10^{18}$ of macromolecules per unit area corresponds to a coiled-coil cross section of $1 \mathrm{~nm}$ as suggested in Ref. [13]. It is important to point out that the only "arbitrary" parameters of the model are the pressure $p$ of the hair cuticula accounting for the protein macromolecules cortex interactions [40] and the shear modulus $\mu$. To the knowledge of the authors, no value for the confinement pressure $p$ is available.

Despite the simplification of the model it quantitatively well reproduces the stiffnesses, the residual stretches, the damage softening, and the sudden softening corresponding to the reconnection to the primary loading curve (return point memory).

In Fig. 5 we reported the cyclic behavior of a spider silk fiber produced by a cellar spider (Pholcus phalangioides). For sample preparation the spider was forced to fall down toward the ground and during its falling it produced its dragline silk, which we collected and glued on a paper frame, resulting in a fiber with $1 \mathrm{~cm}$ length and a diameter of about $1 \mu \mathrm{m}$. The large toughness and healing properties observed in the macroscopic material behavior is the result of a complex multiscale structure and energy exchange phenomena (prestretch, chains alignment, chain-to-chain, and chainsmatrix interactions), here modeled through our additivity assumption.

We point out that the behavior at small stretches is typically regulated by both the amorphous fraction and the tertiary structure [34,44], not considered in our model, so that we reproduced the only path with a previous stretch larger than

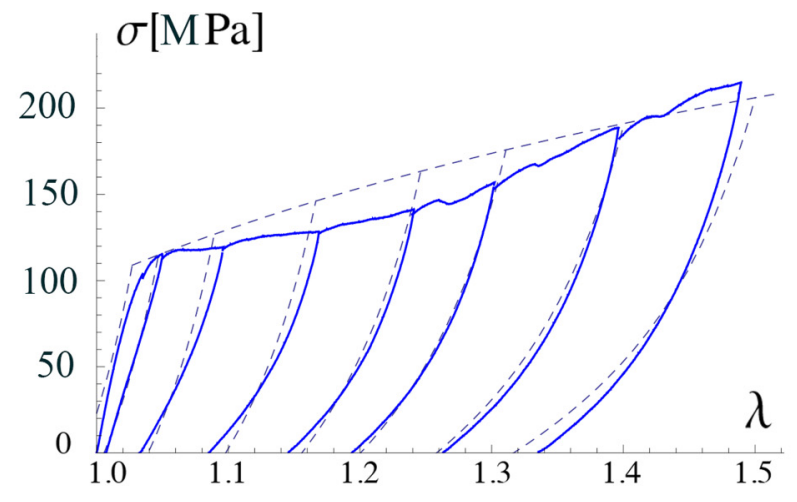

(a)
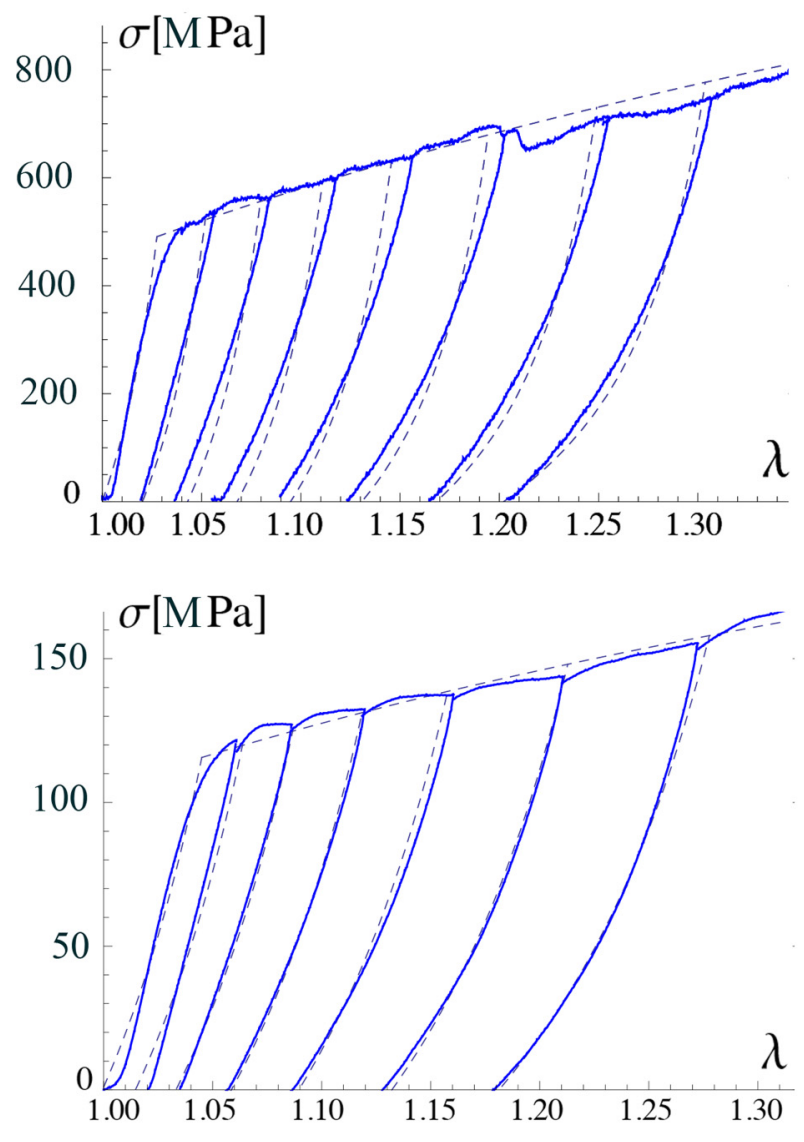

FIG. 4. Cyclic tests on human (a), rabbit (b), and cow (c) hair at room temperature and at a strain rate of $0.1 \mathrm{~s}^{-1}$ (experimental behavior-continuous lines, theoretical model-dashed lines). For the theoretical model we used in (a) for human hair $l_{p}=$ $0.4 \mathrm{~nm}, T=300 \mathrm{~K}, q=Q / l_{c}=45 k_{B} T / \mathrm{nm}, \mu=44 \mathrm{MPa}, p=$ $-130 \mathrm{MPa}, N^{\mathrm{fib}}=10^{18}$; in (b) for rabbit hair $l_{p}=0.45 \mathrm{~nm} ; T=$ $300 \mathrm{~K}, q=Q / l_{c}=180 k_{B} T / \mathrm{nm}, \mu=280 \mathrm{MPa}, p=-375 \mathrm{MPa}$, $N^{\mathrm{fib}}=10^{18}$; in (c) for cow hair $l_{p}=0.33 \mathrm{~nm} ; T=300 \mathrm{~K}, q=$ $Q / l_{c}=45 k_{B} T / \mathrm{nm}, \mu=200 \mathrm{MPa}, p=-130 \mathrm{MPa}, N^{\mathrm{fib}}=10^{18}$.

3\%. Despite its oversimplification, since here we are focused on the main dissipative phenomena, Fig. 5 shows that, even if with lower precision, the model can capture the fundamental phenomena of damage and residual stretches in the case of spider silk, too.

For the pressure we assumed $p=-400 \mathrm{MPa}$ corresponding to an initial prestretch of the fibers of about $25 \%$ in 


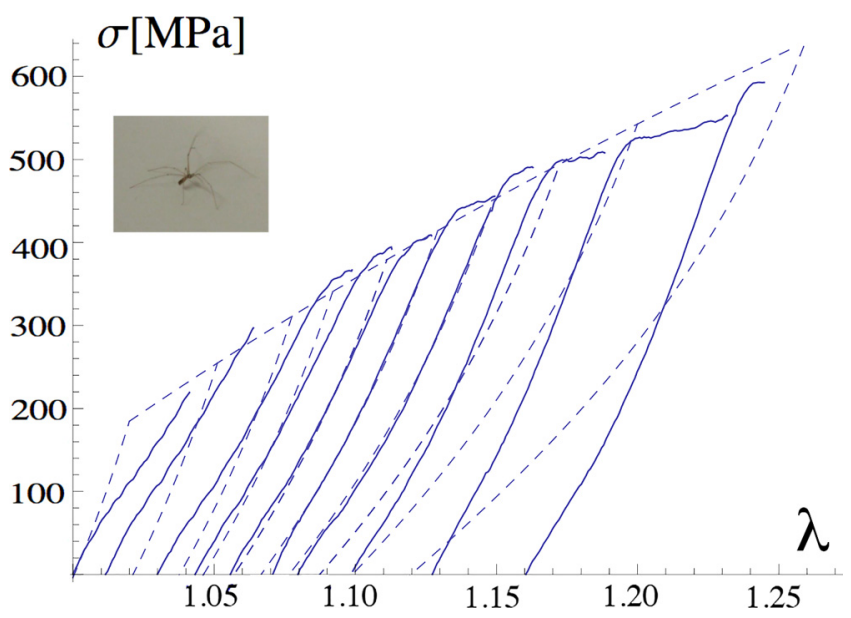

FIG. 5. The dragline silk sample underwent loading and unloading cycles tested through a nanotensile machine (Agilent UTM 150) at room temperature and at a strain rate of $0.1 \mathrm{~s}^{-1}$ (experimental behavior-continuous lines, theoretical model-dashed lines). Here we assumed $l_{p}=0.78 \mathrm{~nm}, l_{c}=25 \mathrm{~nm}, N^{\mathrm{fib}}=4.75 \times 10^{18} \mathrm{~m}^{-2}, Q=$ $550 k_{B} T, T=300 \mathrm{~K}, \mu=670 \mathrm{MPa}, p=-400 \mathrm{MPa}$.

accordance with the experimental evidences in Refs. [32,45]. It is crucial to point out that for the spider fibril response the parameters are chosen to agree with the experimental values. In fact we considered the values $l_{p}=0.8 \mathrm{~nm}$ and $l_{c}=25 \mathrm{~nm}$ estimated in Refs. [32,45] and [14], the unfolding energy $Q=550 k_{B} T$ agrees with the values estimated in Ref. [11]; the number of fibers per unit area $N^{\text {fib }}=4.75 \times 10^{18}$ (per square meters) reflects the results in Refs. [42,46].

Our experimental comparison shows that it is possible to model with remarkable precision the complex mechanical behavior of different protein materials starting from known molecular properties. This represents in our opinion an important step forward in the fundamental comprehension of the link between the properties of protein secondary structure and the macroscopic material properties (toughness, stiffness and large deformations prior to fracture, residual stretches, memory, etc.), which can be of paramount importance for better understanding protein materials and also for the design of new smart bioinspired materials.

\section{ACKNOWLEDGMENTS}

N.M.P. is supported by the European Research Council PoC 2015 "Silkene" No. 693670, by the European Commission H2020 under the Graphene Flagship Core 1 No. 696656 (WP14 "Polymer Composites"), and FET Proactive "Neurofibre" Grant No. 732344.
[1] M. J. Buehler, J. Mech. Mat. Struct. 2, 1019 (2007).

[2] P. Egan, R. Sinko, P. R. LeDuc, and S. Keten, Nat. Commun. 6, 7418 (2015).

[3] G. E. Fantner et al., Biophysical J. 90, 1411 (2006).

[4] R. O. Ritchie, Nat. Mater. 10, 817 (2011).

[5] D. E. Makarov, Individual Proteins Under Mechanical Stress: Lessons from Theory and Computer Simulations, in Singlemolecule Studies of Proteins, edited by Andres F. Oberhauser (Springer, New York, 2013).

[6] S. Keten, Z. Xu, B. Ihle, M. J. Buehler, Nat. Mater. 9, 359 (2010).

[7] J. Chung, A. M. Kushner, A. C. Weisman, and Z. Guan, Nat. Mater. 13, 1055 (2014).

[8] M. Rubinstein and R. H. Colby, Polymer Physics (Oxford University Press, Oxford, 2003).

[9] M. C. Wang and E. Guth, J. Chem. Phys. 20, 1144 (1952).

[10] S. Keten and M. J. Buehler, Phys. Rev. Lett. 100, 198301 (2008).

[11] D. De Tommasi, N. Millardi, G. Puglisi, and G. Saccomandi, J. R. Soc. Interface 10, 20130651 (2013).

[12] H. M. Zbiba and T. D. de la Rubia, Int. J. Plasticity 18, 1133 (2002).

[13] I. Schwaiger, C. Sattler, D. R. Hostetter, and M. Rief, Nat. Mater. 1, 232 (2001).

[14] E. Oroudjev et al., Proc. Natl. Acad. Sci. USA 99, 6460 (2002).

[15] M. Cetinkaya, S. Xiao, B. Markert, W. Stacklies, and F. Gräter, Biophys J. 100, 1298 (2011).

[16] T. Giesa, M. Arslan, N. M. Pugno, and M. J. Buehler, Nano Lett. 11, 5038 (2011).

[17] V. Vogel and M. Sheetz, Nature Rev. Molec. Cell Biol. 7, 265 (2006).

[18] D. J. Selkoe, Nature 426, 900 (2003).
[19] N. Du, X. Y. Liu, J. Narayanan, L. Li, M. L. M. Lim, and D. Q. Li, Biophys. J. 91, 4528 (2006).

[20] J. M. Gosline, P. A. Guerette, C. S. Ortlepp, and K. N. Savage, J. Exp. Biology 202, 3295 (1999).

[21] C. Zhou et al., Biomacromolecules 7, 2415 (2006).

[22] S. M. Liff, N. Kumar, and G. H. Mckinley, Nat. Mater. 6, 76 (2007).

[23] C. Wang, R. J. Stewart, and J. Kopeček, Nature 397, 417 (1999).

[24] J. Kopeček and J. Yang, Acta Biomaterialia 5, 805 (2009).

[25] M. J. Buehler and Y. C. Yung, Nat. Mater. 8, 175 (2009).

[26] J. W. S. Hearle, Int. J. Biol. Macromol. 27, 123 (2000).

[27] Z. Qin and M. J. Buehler, Phys. Rev. Lett. 104, 198304 (2010).

[28] P. J. Flory and B. Erman, Macromolecules 15, 800 (1982).

[29] R. L. C. Akkermans and P. B. Warren, Philos. Trans. R. Soc. London A 362, 1783 (2004).

[30] A. Sponner et al., PLoS ONE 2, e998 (2007).

[31] Y. Liu, Z. Shao, and F. Vollrath, Nat. Mater. 4, 901 (2005).

[32] P. Papadopoulos, J. Sölter, and F. Kremer, Coll. Polym. Sci. 287, 231 (2009).

[33] D. De Tommasi, G. Puglisi, and G. Saccomandi, J. Mech. Phys. Sol. 78, 154 (2015).

[34] M. Rief, M. Gautel et al., Science 276, 1109 (1997).

[35] Y. Y. Termonia, Macromolecules 27, 7378 (1994).

[36] G. Puglisi and L. Truskinovsky, Phys. Rev. E 87, 032714 (2013).

[37] G. Puglisi and L. Truskinovsky, J. Mech. Phys. Sol. 53, 655 (2005).

[38] F. Maddalena, D. Percivale, G. Puglisi, and L. Truskinovsky, Cont. Mech. Therm. 21, 251 (2009).

[39] D. De Tommasi, G. Puglisi, and G. Saccomandi, Biophys. J. 98, 1941 (2010). 
[40] J. Hsin, J. Strümpfer, E. H. Lee, and K. Schulten, Annu. Rev. Biophys. 40, 187 (2011).

[41] V. Stanić, J. Bettini, F. E. Montoro, A. Stein, and K. EvansLutterodt, Nat. Sci. Rep. 5, 17347 (2015).

[42] S. Keten and M. Buehler, Appl. Phys. Lett. 96, 153701 (2010).
[43] S. W. Cranford, J. R. Soc. Interface 10, 20130148 (2013).

[44] A. Nova et al., Nano Lett. 10, 2626 (2010).

[45] R. Ene, P. Papadopoulos, and F. Kremer, Soft Matter 5, 4568 (2009).

[46] C. Riekel and F. Vollrath, Int. J. Biol. Macromol. 29, 203 (2001). 